\title{
Perioperative complications of abdominal surgery in smokers
}

\author{
Yoshikazu Inoue ${ }^{1,2}\left(\right.$ Takahiko Katoh $^{2} \cdot$ Shota Masuda $^{2} \cdot \mathrm{Xi} \mathrm{Lu}^{2} \cdot$ Tadashi Koga $^{1} \cdot$ Tomohiro Sadohara $^{1}$. \\ Michiaki Sadanaga' ${ }^{1}$ Eiji Tanaka ${ }^{3}$
}

Received: 23 December 2019 / Accepted: 12 June 2020 / Published online: 23 June 2020

(C) The Author(s) 2020

\begin{abstract}
Purpose This study examined the association between smoking and perioperative complications of laparoscopic abdominal surgery and whether these complications were reduced with $\geq 4$ weeks of preoperative smoking cessation.

Methods A total of 555 patients who underwent gastric and colorectal cancer surgeries under general anesthesia were divided into the following groups retrospectively: 290 individuals without smoking history (NS group), 144 previous smokers (stopped smoking more than 8 weeks before surgery, PS group), and 121 current smokers (CS group) divided to two groups according to preoperative smoking cessation for $<4$ (CS1, $n=76)$ and $4-8$ weeks (CS2, $n=45)$.

Results When compared with the NS group, postoperative hospitalization duration was significantly longer in the CS1 group $(p<0.01)$, whereas differences between the CS2 or PS groups and NS group were not significant. The total number of postoperative complications was higher in all groups of smoking than in NS group, independent on preoperative smoking cessation; however, suture failure was significantly more frequent only in CS1 group. Although pack-years did not significantly affect complication rates in smokers, duration of smoking cessation time in PS group was a negative predictor of postoperative complications.

Conclusion Providing more than 4 weeks of smoking cessation before gastrointestinal surgery can reduce the duration of hospitalization and rate of suture failure.
\end{abstract}

Keywords Smoking cessation $\cdot$ Postoperative complications $\cdot$ Abdominal surgery

\section{Introduction}

Previous research has identified smoking as an obvious cause of malignant diseases, such as laryngeal, esophageal, and lung cancer; cardiovascular disorders; and diabetes [1-3]. Apart from primary disease, cancer patients who smoke also experience systemic diseases caused by smoking, and considerable effects of smoking on surgery. Previous studies have emphasized smoking as a risk factor for different kinds

Yoshikazu Inoue

yoshikazu9999jpjp@yahoo.co.jp

1 Department of Anesthesiology, Japanese Red Cross Kumamoto Hospital, 2-1-1 Nagamineminami, Higashi-ku, Kumamoto 861-8528, Japan

2 Department of Public Health, Faculty of Life Sciences, Kumamoto University, 1-1-1 Honjou, Chuo-ku, Kumamoto 860-8556, Japan

3 Department of Surgery, Japanese Red Cross Kumamoto Hospital, 2-1-1 Nagamineminami, Higashi-ku, Kumamoto 861-8528, Japan of postoperative complications in various types of patients and surgeries, including gastrointestinal surgery [4-8]. Interventions of preoperative smoking cessation reduced postoperative complications in different kinds of patients [7-13]. In some studies, the proportions of surgeons, anesthesiologists abroad [14], thoracic surgeons, and anesthesiologists in Japan [15] who actively provided preoperative smoking cessation guidance were approximately $58 \%, 30 \%, 26 \%$, and $6 \%$, respectively; hence, it is recommended to further highlight the benefits of this approach.

Although most studies showed positive effects of preoperative smoking cessation, some did not find significantly improved outcomes [16, 17]. These inconsistencies likely reflect the differences in duration of preoperative smoking cessation, variability in inclusion criteria (different patients, different types of surgery) and different endpoints (all complications, special type of complications, mortality). In this context, it is still unclear how long period of smoking cessation before gastrointestinal surgery is needed to reduce postoperative complications and reduce hospital 
stays. The minimum reported beneficial durations of smoking cessation before surgery were 2 weeks [8], 3 weeks [18], 4 weeks [19], or 6-8 weeks [20]. In gastric cancer surgery, at least 2 weeks of smoking cessation [8] helped reduce the incidence of postoperative complications, whereas another study [7] reported lower postoperative pulmonary problems in patients who stopped smoking $\geq 4$ weeks before surgery. Meanwhile, for patients undergoing colorectal surgery, short-term cessation (2-3 weeks) failed to improve tissue and wound healing or avoid other complications [17].

Here, we hypothesized that rates of postoperative complications and duration of hospital stays decline with longer smoking cessation times before surgery. The aim of this study was to compare the postoperative complication rate and duration of postoperative hospital stays (as primary endpoints) between patients who stopped smoking for different time periods before the surgery (less than 4 weeks, 4-8 weeks, and $>8$ weeks) and patients who never smoked. The secondary objective was to assess the effects of packyears and exact smoking cessation duration on complication rates.

\section{Methods}

In this retrospective, observational cohort study, we collected clinical data of patients with the American Society of Anesthesia physical status classification of $1 / 2$, who had undergone laparoscopic abdominal surgery for gastric and colon cancers. The study was conducted at Kumamoto Red Cross Society, a regional hospital in Japan. The institutional ethics committee approved the study protocol (\#268), and patient confidentiality was maintained throughout the study. Opt-out was used because it was an observational study in accordance with ethical guidelines for humans.

All patients who had undergone gastric and colorectal cancer laparoscopic surgeries under general anesthesia between January 2015 and December 2019 were considered $(n=622)$; among them, 45 cases with resection of gallbladder, uterine appendage, or double tumor, radiation therapy before surgery, or metastasis, and 22 patients lacking preoperative smoking information were excluded. Finally, 555 cases were included in the study. We divided the participants into four groups according to smoking status at the time of operation: patients who stopped smoking $<4$ weeks before the surgery (CS1, $n=76$ ), those with smoking cessation $4-8$ weeks before the surgery (CS2, $n=45$ ), those with smoking cessation $>8$ weeks before the surgery (PS group, $n=144$ ), and those who never smoked (NS group, $n=290$ ) (Fig. 1). Age, sex, body mass index, FEV1/FVC ratio, time under anesthesia, bleeding volume, incidence of postoperative complications, and postoperative hospitalization duration were compared among these groups. The primary endpoints were the incidence of postoperative

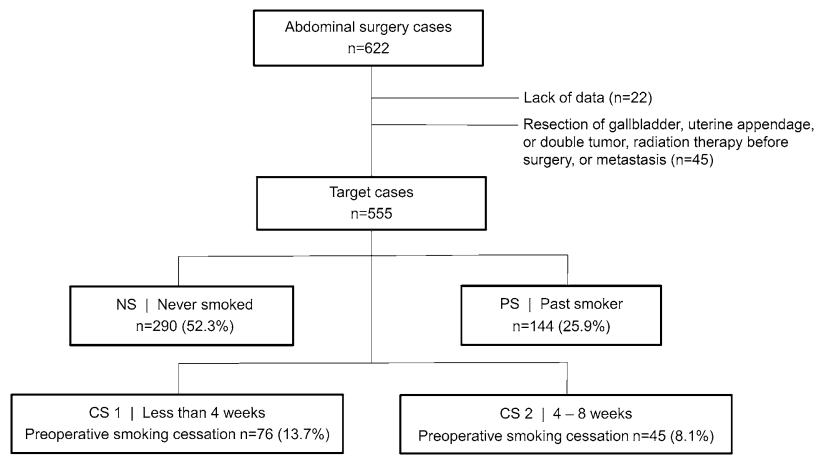

Fig. 1 Flowchart of the study

complications and duration of postoperative hospital stay. Secondary endpoints were correlations between the pack-years and occurrence of complications in all smoking groups, and between smoking cessation period and complications in the PS group.

Postoperative complications were recorded in accordance to Clavien-Dindo classification of postoperative complications proposed by Dindo et al. [21]. Specifically, we recorded complications with grade II or higher, which involve complications requiring postoperative medication other than antiemetics, antipyretics, analgesics, and diuretics. Postoperative complications were divided into respiratory (pneumonia, pleural effusion, and atelectasis); digestive tract (suture failure and intestinal obstruction); circulatory (arrhythmia, thrombosis, and angina pectoris), surgical wound (infection); and other (delirium, urinary tract infections, and unknown fever) complications. The complications were recorded from the medical records and were based on the clinical, biochemical, and/ or radiological manifestations. We considered postoperative complications and site, presence or absence of diabetes, and alcohol consumption as potential confounding variables.

Statistical analyses were performed using EZR version 2.4-0 (Saitama Medical Center, Jichi Medical University, Saitama, Japan [22])—a graphical user interface for R (The R Foundation for Statistical Computing, Vienna, Austria). Continuous data were expressed as means and standard deviations. Student's $t$ test was used to compare continuous variables and chi-square test to compare categorical variables. Inter-group comparisons were performed using one-way ANOVA, and Tukey's post hoc test was used for multiple comparisons. Adjusted odds ratios were calculated using multivariate logistic regression to control for potential confounders. Statistical significance was set at $p<0.01$. 


\section{Results}

Among 555 patients, 450 (81.1\%) used inhaled anesthetics and $105(18.9 \%)$ underwent general anesthesia with TIVA. Epidural anesthesia was not used in 26 cases (4.7\%). In patients who did not use epidural anesthesia for postoperative analgesia, continuous infusion of fentanyl citrate was performed after surgery. Gastric cancer surgery was performed by nine surgeons [one surgeon performed surgeries in 155/191 (81.2\%) cases]. Colorectal cancer surgery was performed by 12 surgeons [two surgeons performed surgeries in 288/364 (79.1\%) cases].

The CS1 group was significantly younger than the PS and NS groups (Table 1). The proportion of men in NS group was significantly lower than in each smoking group, while CS1 group additionally showed lower percent of men as compared to CS2 and PS groups (Table 1). The average FEV1/FVC ratios were significantly lower in each smoking group compared to the NS group ( $p<0.01$; Table 1$)$. Total preoperative complications, surgical time, anesthesia time, and bleeding volume did not differ significantly among the groups. However, incidence of postoperative complications was significantly higher in the CS1 and PS groups than in the NS group ( $p<0.01$; Table 1). Postoperative hospitalization duration was significantly longer in the CS1 group compared with NS group $(p<0.01$; Table 1). Logistic regression analysis showed that the odds for all kinds of complications were affected by smoking ( $p<0.01$; Table 2$)$. When considering only suture failure
Table 1 Basic demographic and clinical characteristics of included patients $(n=555)$

\begin{tabular}{|c|c|c|c|c|c|}
\hline \multirow[t]{2}{*}{ Variable } & \multicolumn{4}{|l|}{ Group } & \multirow[t]{2}{*}{$P$ value } \\
\hline & $\mathrm{NS}(n=290)$ & $\operatorname{CS} 1(n=76)$ & $\operatorname{CS} 2(n=45)$ & PS $(n=144)$ & \\
\hline Age (years) & $70.4 \pm 10.9$ & $64.9 \pm 11.5$ & $65.9 \pm 10.6$ & $69.5 \pm 10.9$ & $<0.001^{*}, \|$ \\
\hline Sex & & & & & $<0.001^{*, \dagger, \ddagger, \S, \|}$ \\
\hline Male & $102(35.2)$ & $80(76.9)$ & $38(84.4)$ & $130(90.3)$ & \\
\hline Female & $188(64.8)$ & $24(23.1)$ & $7(15.6)$ & $14(9.7)$ & \\
\hline Body mass index & $23.0 \pm 4.0$ & $22.6 \pm 3.1$ & $23.4 \pm 3.1$ & $23.6 \pm 3.9$ & 0.373 \\
\hline Pack-years & I & $36.3 \pm 18.2$ & $41.2 \pm 23.3$ & $34.3 \pm 22.6$ & 0.103 \\
\hline Preoperative complications & $203(70.1)$ & $53(69.7)$ & $31(68.9)$ & 109 (75.7) & 0.631 \\
\hline HT & $106(52.2)$ & $14(26.4)$ & $13(41.9)$ & $47(43.1)$ & $<0.01^{*}$ \\
\hline $\mathrm{DM}$ & $16(7.9)$ & $6(11.3)$ & $5(16.1)$ & $9(8.3)$ & 0.405 \\
\hline $\mathrm{HT}+\mathrm{DM}$ & $33(16.3)$ & $14(26.4)$ & $4(12.9)$ & $21(19.3)$ & 0.326 \\
\hline COPD & $0(0)$ & $3(5.7)$ & $1(3.2)$ & $5(4.6)$ & 0.014 \\
\hline FEV1.0 / FVC (\%) & $81.3 \pm 6.4$ & $77.3 \pm 7.9$ & $76.8 \pm 7.9$ & $77.4 \pm 5.6$ & $<0.001^{*, \dagger}$, ま \\
\hline Surgical time (min) & $271 \pm 83$ & $284 \pm 122$ & $296 \pm 73$ & $285 \pm 94$ & 0.291 \\
\hline Anesthesia time (min) & $328 \pm 85$ & $345 \pm 126$ & $349 \pm 73$ & $346 \pm 97$ & 0.230 \\
\hline Bleeding volume (ml) & $100 \pm 254$ & $84 \pm 151$ & $135 \pm 258$ & $93 \pm 217$ & 0.803 \\
\hline Postoperative complications & $17(5.9)$ & $21(27.6)$ & $7(15.5)$ & $29(20.1)$ & $<0.001^{*} \ddagger$ \\
\hline Suture failure & $4(1.4)$ & $8(10.5)$ & $1(2.2)$ & $3(2.1)$ & $<0.01 *$ \\
\hline Other complications & $13(4.5)$ & $13(17.1)$ & $6(13.3)$ & $26(18.0)$ & $<0.001^{*}, \stackrel{+}{+}$ \\
\hline Intestinal obstruction & $4(23.5)$ & $1(4.8)$ & $4(57.1)$ & $6(20.7)$ & \\
\hline Pneumonia & $2(11.8)$ & $2(9.5)$ & $0(0)$ & $3(10.3)$ & \\
\hline Arrhythmia & $0(0)$ & $1(4.8)$ & $0(0)$ & $2(6.9)$ & \\
\hline $\begin{array}{l}\text { Postoperative hospitaliza- } \\
\text { tion duration (days) }\end{array}$ & $9.5 \pm 4.0$ & $12.2 \pm 10.9$ & $11.6 \pm 5.9$ & $11.3 \pm 7.5$ & $<0.01 *$ \\
\hline
\end{tabular}

Values are presented as $n(\%)$ or mean $\pm \mathrm{SD}$

$N S$ patients without smoking history, CS 1- current smokers who stopped smoking less than 4 weeks prior to surgery, CS 2- current smokers who stopped smoking 4-8 weeks prior to surgery, PS past smokers (stopped smoking more than 8 weeks prior to surgery); Preoperative complications (obtained from the description in the medical records): $H T$ hypertension, $D M$ diabetes mellitus, COPD chronic obstructive pulmonary disease, others (e.g., bronchial asthma, chronic kidney disease, atrial fibrillation)

$F E V 1.0$ forced expiratory volume in one second, $F V C$ forced vital capacity

Postoperative complications were recorded as grade II or higher in Clavien-Dindo classification of postoperative complications [21]

Significant post hoc pairwise comparisons $(P<0.05)$

*NS versus $\mathrm{CS} 1,{ }^{\dagger} \mathrm{NS}$ versus $\mathrm{CS} 2,{ }^{\dagger} \mathrm{NS}$ versus PS, ${ }^{\S} \mathrm{CS} 1$ versus $\mathrm{CS} 2,{ }^{\circ} \mathrm{CS} 1$ versus PS 
Table 2 Odds ratios (95\% confidence intervals for OR) for the presence of postoperative complications depending on the smoking status and confounding variables

\begin{tabular}{lllll}
\hline Predictor variable & Complications & \multicolumn{2}{l}{ Groups } & \\
\cline { 3 - 5 } & & CS $1(n=76)$ & CS 2 $(n=45)$ & PS $(n=144)$ \\
\hline Smoking status & All complications & $5.54[2.73-11.2]^{*}$ & $4.01[1.67-9.67]^{*}$ & $3.89[2.01-7.55]^{*}$ \\
& Suture failure & $8.17[2.39-27.9]^{*}$ & $3.33[0.59-18.7]$ & $1.79[0.39-8.11]$ \\
Smoking status adjusted $^{\#}$ & All complications & $7.53[3.08-18.7]^{*}$ & $5.95[1.99-17.8]^{*}$ & $5.30[2.28-12.3]^{*}$ \\
& Suture failure & $8.83[1.55-50.3]^{*}$ & $5.78[0.66-50.4]$ & $2.25[0.31-16.5]$ \\
\hline
\end{tabular}

\#Adjusted for age, diabetes, alcohol, anesthesia time, and surgical site

CS 1- current smokers who stopped smoking less than 4 weeks prior to surgery, CS 2-current smokers who stopped smoking 4-8 weeks prior to surgery, $P S$ past smokers (stopped smoking more than 8 weeks prior to surgery)

$* p<0.01$ as complications, only the CS1 group showed significantly more complications, both before and after adjusting for age, diabetes, alcohol consumption, anesthesia time, and surgical site (Table 2).

The pack-years of each smoking group are shown in Table 1. The presence of complications did not depend on pack-years in any group ( $p>0.05$ for all). In the PS group, smoking cessation period was $17.9 \pm 13.3$ years, and there was significant negative correlation between the presence of complications and duration of smoking cessation until surgery $(p<0.01)$ (Table 3$)$.

\section{Discussion}

In this study we explored whether preoperative smoking cessation was able to reduce postoperative complications after laparoscopic gastrointestinal surgery. In contrast to those who stopped smoking $<4$ weeks before surgery, individuals who stopped smoking more than four weeks before the surgery had comparable duration of hospitalization and number of suture failure complications as non-smokers.

Patient's age was significantly lower in the group with $<4$ weeks smoking cessation compared to PS and NS groups, suggesting that cancer occurred at significantly younger age. Smoking is a potential risk factor for gastric and colon cancers. FEV1/FVC ratio was significantly higher in the NS than in CS1, CS2, and PS groups, likely reflecting that smokers have first signs of obstructive pulmonary diseases, such as pulmonary emphysema. All smoking groups showed higher occurrence of all types of complications than NS group, which corresponds to previous studies regarding digestive surgery that showed increased frequency of respiratory complications and postoperative suture defects in smokers $[23,24]$ and likely adverse effects on wound healing. Indeed, smoking is an independent risk factor for postoperative suture failure [4-6] because of impaired blood flow at the anastomotic site, which occurs because of vasoconstrictive action of tobacco smoke including nicotine [25, 26]; this could explain many suture failure cases. In contrast to Jung et al. where even preoperative smoking cessation for at least 2 weeks helped to reduce postoperative complications in gastric cancer surgery [8], we observed no improvements under 4 weeks. In another study in gastric cancer patients, 4 weeks of smoking cessation were sufficient to reduce pulmonary complications [7]. However, in our study, considering all postoperative complications, all three smoking groups had more complications than the NS group, suggesting that even after longer smoking cessation some of the negative effects of smoking remain, predisposing to some of the complications. However, statistical significance was achieved only for the CS1 vs. NS group, and for the PS vs. NS group comparisons, whereas comparisons of CS2 with NS or PS were not significant. Lack
Table 3 Odds ratios (95\% Confidence Intervals for OR) for the presence of postoperative complications based on the pack-years and duration of smoking cessation

\begin{tabular}{llll}
\hline Predictor variable & Group & \\
\cline { 2 - 4 } & CS 1 $(n=76)$ & CS 2 $(n=45)$ & PS $(n=144)$ \\
\hline Pack-years & $1.00[0.98-1.02]$ & $0.98[0.93-1.02]$ & $1.01[0.99-1.03]$ \\
$\begin{array}{l}\text { Duration of smoking cessa- } \\
\text { tion (years) }\end{array}$ & - & - & $0.93[0.88-0.97]^{*}$ \\
\hline
\end{tabular}

CS 1- current smokers who stopped smoking less than 4 weeks prior to surgery, CS 2-current smokers who stopped smoking 4-8 weeks prior to surgery, $P S$-past smokers (stopped smoking more than 8 weeks prior to surgery)

$* p<0.01$ 
of significant differences in postoperative complications between CS2 and NS groups, and CS2 and PS groups, may stem from a relatively small size of CS2 group. Considering a large standard deviation in smoking cessation period for the PS group (17.9 \pm 13.3 years), some of those patients had a smoke-free period close to that in CS2 group, additionally contributing to lack of differences. Furthermore, negative correlation between the presence of complications and duration of smoking cessation before surgery in the PS group suggests that smoking cessation before surgery may be more important than pack-years in reducing postoperative complications. Although postoperative complications were significantly higher in the PS compared with NS group, it was evident that the PS group actually showed comparable number of typical postoperative complications, but a higher number of other complications (Table 1). Hence, the definition of postoperative complications should be considered when interpreting the results. In addition, although not significantly different, the higher rate of preoperative complications in the PS group may have also contributed to its increased postoperative complications.

Nevertheless, if focusing on suture failures alone, while the CS1 group had significantly more suture failure cases than NS group, these complications were not more common in the CS2 and PS groups than in NS group. These results suggested that more than four weeks of smoking cessation can reduce the rate of suture failure. Our results support the previous findings [17] on inability of short-term (2-3 weeks) cessation of smoking to reduce the risk of complicated tissue and wound healing in colorectal surgery. High overall postoperative complications in the CS and PS groups are not inconsistent with previous studies, although we did not expect this high number of complications. Therefore, smoking should be prohibited to avoid additional increase in complications.

Duration of hospitalization was significantly longer in CS1 group when compared with NS group, but CS2 and PS groups had intermediate durations between the CS1 and NS, without significant differences from the NS group. These findings indicate that $>4$ weeks of smoking cessation before surgery contributes to shorter hospitalization times as opposed less than 4 weeks before surgery. However, some studies have demonstrated the effectiveness of short-term smoking cessation [27, 28]. Previous research showed that respectively $\sim 8$ and 4 weeks of smoking cessation were required to reduce postoperative respiratory complications after coronary artery bypass surgery [29] and lung resection $[30,31]$. In breast reconstruction surgery, preoperative smoking cessation of at least 3 weeks resulted in a similar overall complication rate to nonsmokers [32]. Wound healing following head and neck reconstruction surgery improved with 3 weeks of preoperative smoking cessation [18]. Furthermore, smoking cessation of $\geq 4$ weeks reduced complications such as skin flap necrosis [33]. These results demonstrate the effectiveness of short-term smoking cessation. However, in gastrointestinal surgery, as in this study, $<4$ weeks of smoking cessation did not reduce complications and hospital stay. Postoperative complications were suggested to decrease with increasing smoking cessation duration [27]. Our study showed that postoperative complications were less likely with longer cessation time among those in the PS group.

Although we did not evaluate mortality rates, previous studies suggested that smoking cessation is advisable to reduce mortality in patients with colon cancer [34-36], and benefits of smoking cessation increase over time [37]. Indeed, we observed that longer smoking cessation duration was associated with fewer complications.

Preoperative smoking cessation could be beneficial, even in gastrointestinal surgery, for which short-term effects of smoking cessation are unclear. Perioperative period is a perfect teachable moment for smoking cessation and provides smokers with the opportunity to cease smoking for long term [38]. In this context, everyone involved in care during the perioperative period is in a position to improve not only short-term surgical outcomes, but also long-term health outcomes and costs. As complications following abdominal surgery are common for both CS and PS groups, it is important to prevent starting smoking in the first place, rather than quitting before surgery.

The study has some limitations. Relatively small number of cases in some groups may have contributed to smaller power to detect some inter-group differences. The preoperative smoking cessation period was confirmed only via interview, while biochemical indicators, such as urinary and salivary cotinine, were not used. Examination of passive smoking and electronic cigarettes was insufficient. We were unable to examine preoperative complications that could affect postoperative complications in detail; however, their effects appeared to be small, because only patients with the American Society of Anesthesia physical status classification of $1 / 2$ were included.

In conclusion, our results showed that although more postoperative complications were observed in CS1, CS2, and PS groups compared to the NS group, only the CS1 group had higher rates of suture failures and longer hospitalization time than NS group. Moreover, the complications decreased significantly with prolonged cessation periods in the PS group. Taken together, our results indicate that some of the complications can be reduced by providing at least 4 weeks of smoking cessation before gastrointestinal surgery.

Acknowledgements We would like to thank Editage (www.edita ge.com) for English language editing. 


\section{Compliance with ethical standards}

Conflict of interest The authors have no conflicts of interest to disclose.

Open Access This article is licensed under a Creative Commons Attribution 4.0 International License, which permits use, sharing, adaptation, distribution and reproduction in any medium or format, as long as you give appropriate credit to the original author(s) and the source, provide a link to the Creative Commons licence, and indicate if changes were made. The images or other third party material in this article are included in the article's Creative Commons licence, unless indicated otherwise in a credit line to the material. If material is not included in the article's Creative Commons licence and your intended use is not permitted by statutory regulation or exceeds the permitted use, you will need to obtain permission directly from the copyright holder. To view a copy of this licence, visit http://creativecommons.org/licenses/by/4.0/.

\section{References}

1. Mannami T, Iso H, Baba S, Sasaki S, Okada K, Konishi M, Tsugane S. Japan Public Health Center-Based Prospective Study on Cancer and Cardiovascular Disease Group. Cigarette smoking and risk of stroke and its subtypes among middle-aged Japanese men and women: the JPHC Study Cohort I. Stroke. 2004;35:1248-53.

2. Huxley R, Jamrozik K, Lam TH, Barzi F, Ansary-Moghaddam A, Jiang CQ, Suh I, Woodward M. Asia pacific cohort studies collaboration. Impact of smoking and smoking cessation on lung cancer mortality in the Asia-Pacific region. Am J Epidemiol. 2007;165:1280-6.

3. Mannino DM, Aguayo SM, Petty TL, Redd SC. Low lung function and incident lung cancer in the United States: Data from the first national health and nutrition examination survey follow-up. Arch Intern Med. 2003;163:1475-80.

4. Bhama AR, Batool F, Collins SD, Ferraro J, Cleary RK. Risk factors for postoperative complications following diverting loop Ileostomy takedown. J Gastrointest Surg. 2017;21:2048-55.

5. Rencuzogullari A, Benlice C, Valente M, Abbas MA, Remzi FH, Gorgun E. Predictors of anastomotic leak in elderly patients after colectomy: Nomogram-based assessment from the american college of surgeons national surgical quality program proceduretargeted cohort. Dis Colon Rectum. 2017;60:527-36.

6. Midura EF, Hanseman D, Davis BR, Atkinson SJ, Abbott DE, Shah SA, Paquette IM. Risk factors and consequences of anastomotic leak after colectomy: a national analysis. Dis Colon Rectum. 2015;58:333-8.

7. Quan H, Ouyang L, Zhou H, Ouyang Y, Xiao H. The effect of preoperative smoking cessation and smoking dose on postoperative complications following radical gastrectomy for gastric cancer: a retrospective study of 2469 patients. World J Surg Oncol. 2019;17:61.

8. Jung KH, Kim SM, Choi MG, Lee JH, Noh JH, Sohn TS, Bae JM, Kim S. Preoperative smoking cessation can reduce postoperative complications in gastric cancer surgery. Gastric Cancer. 2015;18:683-90.

9. Bluman LG, Mosca L, Newman N, Simon DG. Preoperative smoking habits and postoperative pulmonary complications. Chest. 1998;113:883-9.

10. Nakagawa M, Tanaka H, Tsukuma H, Kishi Y. Association between the duration of the preoperative smoke-free period and the incidence of postoperative pulmonary complications after pulmonary surgery. Chest. 2001;120:705-10.
11. Turan A, Mascha EJ, Roberman D, Turner PL, You J, Kurz A, Sessler DI, Saager L. Smoking and perioperative outcomes. Anesthesiology. 2011;114:837-46.

12. Warner MA, Divertie MB, Tinker JH. Preoperative cessation of smoking and pulmonary complications in coronary artery bypass patients. Anesthesiology. 1984;60:380-3.

13. Warner MA, Offord KP, Warner ME, Lennon RL, Conover MA, Jansson-Schumacher U. Role of perioperative cessation of smoking and other factors in postoperative pulmonary complications: A blinded prospective study of coronary artery bypass patients. Mayo Clin Proc. 1989;64:609-16.

14. Warner DO, Sarr MG, Offord KP, Dale LC. Anesthesiologists, general surgeons, and tobacco interventions in the perioperative period. Anesth Analg. 2004;99:1766-73.

15. Kai T, Maki T, Takahashi S, Warner DO. Perioperative tobacco use interventions in Japan: A survey of thoracic surgeons and anaesthesiologists. Br J Anaesth. 2008;100:404-10.

16. Rodriguez M, Gomez-Hernandez MT, Novoa N, Jimenez MF, Aranda JL, Varela G. Refraining from smoking shortly before lobectomy has no influence on the risk of pulmonary complications: a case-control study on a matched population. Eur J Cardiothoracic Surg. 2016;51:498-503.

17. Sørensen LT, Jørgensen T. Short-term pre-operative smoking cessation intervention does not affect postoperative complications in colorectal surgery: a randomized clinical trial. Colorectal Dis. 2003;5:347-52.

18. Kuri M, Nakagawa M, Tanaka H, Hasuo S, Kishi Y. Determination of the duration of preoperative smoking cessation to improve wound healing after head and neck surgery. Anesthesiology. 2005;102:892-6.

19. Lindström D, Azodi OS, Wladis A, Tønnesen H, Linder S, Nåsell $\mathrm{H}$, Ponzer S, Adami J. Effects of a perioperative smoking cessation intervention on postoperative complications: a randomized trial. Ann Surg. 2008;248:739-45.

20. Møller AM, Villebro N, Pedersen T, Tønnesen H. Effect of preoperative smoking intervention on postoperative complications: a randomised clinical trial. Lancet. 2002;359:114-7.

21. Dindo D, Demartines N, Clavien PA. Classification of surgical complications: a new proposal with evaluation in a cohort of 6336 patients and results of a survey. Ann Surg. 2004;240:205-13.

22. Kanda Y. Investigation of the freely available easy-to-use software 'EZR' for medical statistics. Bone Marrow Transplant. 2013;48:452-8.

23. Taflampas P, Christodoulakis M, Tsiftsis DD. Anastomotic leakage after low anterior resection for rectal cancer: Facts, obscurity, and fiction. Surg Today. 2009;39:183-8.

24. Cooke DT, Lin GC, Lau CL, Zhang L, Si MS, Lee J, Chang AC, Pickens A, Orringer MB. Analysis of cervical esophagogastric anastomotic leaks after transhiatal esophagectomy: Risk factors, presentation, and detection. Ann Thorac Surg. 2009;88:177-85.

25. Araco A, Gravante G, Sorge R, Araco F, Delogu D, Cervelli $\mathrm{V}$. Wound infections in aesthetic abdominoplasties: The role of smoking. Plast Reconstr Surg. 2008;121:305e-e310310.

26. Black CE, Huang N, Neligan PC, Levine RH, Lipa JE, Lintlop S, Forrest CR, Pang CY. Effect of nicotine on vasoconstrictor and vasodilator responses in human skin vasculature. Am J Physiol Regul Integr Comp Physiol. 2001;281:R1097-R11041104.

27. Mills E, Eyawo O, Lockhart I, Kelly S, Wu P, Ebbert JO. Smoking cessation reduces postoperative complications: A systemic review and meta-analysis. Am J Med. 2011;124:144-54.

28. Myers K, Hajek P, McRobbie H. Stopping smoking shortly before surgery and postoperative complications: A systematic review and meta-analysis. Arch Intern Med. 2011;171:983-9.

29. Warner MA, Offord KP, Warner ME, Lennon RL, Conover MA, Jansson-Schumacher U. Role of preoperative cessation of smoking and other factors in postoperative pulmonary complications: 
A blinded prospective study of coronary artery bypass patients. Mayo Clin Proc. 1989;64:609-16.

30. Barrera R, Shi W, Amar D, Thaler HT, Gabovich N, Bains MS, White DA. Smoking and timing of cessation: Impact on pulmonary complications after thoracotomy. Chest. 2005;127:1977-83.

31. Vaporciyan AA, Merriman KW, Ece F, Roth JA, Smythe WR, Swisher SG, Walsh GL, Nesbitt JC, Putnam JB Jr. Incidence of major pulmonary morbidity after pneumonectomy: Association with timing of smoking cessation. Ann Thorac Surg. 2002;73:420-6.

32. Padubidri AN, Yetman R, Browne E, Lucas A, Papay F, Larive B, Zins J. Complications of postmastectomy breast reconstructions in smokers, ex-smokers, and nonsmokers. Plast Reconstr Surg. 2001;107:342-9.

33. Chang DW, Reece GP, Wang B, Robb GL, Miller MJ, Evans GR, Langstein HN, Kroll SS. Effect of smoking on complications in patients undergoing free TRAM flap breast reconstruction. Plast Reconstr Sur. 2000;105:2374-80.

34. Yang B, Jacobs EJ, Gapstur SM, Stevens V, Campbell PT. Active smoking and mortality among colorectal cancer survivors: The Cancer Prevention Study II nutrition cohort. J Clin Oncol. 2015;33:885-93.

35. Ordóñez-Me JM, Walter V, Schöttker B. Jenab M, O’Doherty MG, Kee F, Bueno-de-Mesquita B, Peeters PHM, Stricker BH, Ruiter R, Hofman A Söderberg S, Jousilahti P, Kuulasmaa K, Freedman ND, Wilsgaard T, Wolk A, Nilsson LM, Tjønneland A, Quirós
JR, van Duijnhoven FJB, Siersema PD, Boffetta P, Trichopoulou A, Brenner H. Consortium on Health and Ageing: Network of Cohorts in Europe and the United States (CHANCES). Impact of prediagnostic smoking and smoking cessation on colorectal cancer prognosis: A meta-analysis of individual patient data from cohorts within the CHANCES consortium. Ann Oncol. 2018;29:472-83

36. Walter V, Jansen L, Hoffmeister M, Ulrich A, Chang-Claude J, Brenner H. Smoking and survival of colorectal cancer patients: Population-based study from Germany. Int J Cancer. 2015;137:1433-45.

37. Mason DP, Subramanian S, Nowicki ER, Grab JD, Murthy SC, Rice TW, Blackstone EH. Impact of smoking cessation before resection of lung cancer: A Society of Thoracic Surgeons General Thoracic Surgery Database study. Ann Thorac Surg. 2009;88:362-70.

38. Yousefzadeh A, Chung F, Wong DT, Warner DO, Wong J. Smoking cessation: the role of the anesthesiologist. Anesth Analg. 2016;122:1311-20.

Publisher's Note Springer Nature remains neutral with regard to jurisdictional claims in published maps and institutional affiliations. 